\title{
A Case Report: Isolated Pontine Lesion in Hypertensive of a Pregnant Patient with Idiopathic Thrombocytopenia
}

\author{
Authors: \\ Ilknur Yardimci, ${ }^{1}$ Mehmet Tunc, ${ }^{1}$ Bahar Say, ${ }^{1}$ Cemile Dayangan Sayan,, \\ Mirace Yasemin Karadeniz Bilgili, ${ }^{3}$ Selim Yalcin, ${ }^{4}{ }^{*}$ Ozlem Coskun ${ }^{1}$ \\ 1. Department of Neurology, Kırıkkale University, Kırıkkale, Turkey \\ 2. Department of Obstetrics and Gynecology, Kırıkkale University, Kırıkkale, Turkey \\ 3. Department of Radiology, Kırıkkale University, Kırıkkale, Turkey \\ 4. Department of Hematology Oncology, Kırıkkale University, Kırıkkale, Turkey \\ *Correspondence to oecoskun@yahoo.com
}

Disclosure: $\quad$ The authors have declared no conflicts of interest.

Received: $\quad 06.12 .19$

Accepted: $\quad 12.02 .20$

Keywords: Isolated pontine lesion, posterior reversible encephalopathy syndrome (PRES), pregnancy, thrombocytopenia, spreading depression.

Citation:

EMJ. 2020;5[2]:103-107.

\section{Abstract}

Posterior reversible encephalopathy syndrome (PRES) is a neuroradiologic diagnosis characterised by headache, seizures, altered mental status, and a spectrum of visual deficits ranging from visual neglect to cortical blindness. PRES manifestation is a situation of medical emergency; however, it is a fully reversible condition, especially when diagnosed and treated immediately. The main problem lies in the impairment of cerebral blood flow autoregulation which, in turn, leads to endothelial dysfunction and vasogenic brain oedema. MRI indicates cerebral oedema in the occipital, temporal, and parietal lobes. Brainstem involvement is very rare in the literature. Idiopathic thrombocytopenia-related PRES is also rare in the literature. In this article, a case of PRES with only pons involvement in MRI after hypertensive attack in a pregnant patient with idiopathic thrombocytopenia is presented. PRES may present only brainstem involvement, as seen in this present case. Whether or not mild hypertension and mild thrombocytopenia found in this case are associated with limited disease should be evaluated. Prolonged spreading depression may have a role in the pathophysiology of PRES.

\section{INTRODUCTION}

Posterior reversible encephalopathy syndrome (PRES) is a clinical condition characterised by headache, visual disturbances, seizures, and confusion. ${ }^{1,2}$ The main problem lies in the impairment of cerebral blood flow autoregulation which, in turn, leads to endothelial dysfunction and vasogenic brain oedema.

PRES is often related to an acute increase in arterial blood pressure, is clinically indistinguishable from hypertensive encephalopathy, and has been associated with pre-eclampsia, renal failure, infections, immunosuppressive agent requirement, and hypercalcaemia. It presents with abnormal findings on CT and MRI indicating cerebral oedema in the occipital, temporal, and parietal lobes. Rarely, the frontal lobe, brainstem, and cerebellum may also be involved, however 
brainstem involvement is very rare., ${ }^{2,3}$ In the literature, the authors found only seven cases with isolated pons involvement in PRES. ${ }^{2-7}$ Spinal cord involvement in PRES was also rarely reported. ${ }^{8}$ Ou et al. ${ }^{4}$ reviewed the literature to identify isolated infratentorial brain involvement. In their study, dissimilar to this present case, the presence of extremely high blood pressure at onset was essential to the development of infratentorial isolated involved PRES. Additionally, in their series $27 \%$ of patients' disease was accompanied by obstructive hydrocephalus, which is not usually seen in typical PRES.?

Herein, a patient with PRES who had thrombocytopenia because of idiopathic thrombocytopenia (ITP) and in whom the pons was solely involved is presented. The authors also reviewed the literature regarding treatment, MRI abnormalities, and risk factors of PRES.

\section{CASE REPORT}

A 24-year-old woman admitted for elective caesarean section at 36 weeks of amenorrhoea. This was her first pregnancy. She had ITP, but had received good prenatal care, with a normal prenatal analysis. On admission, her blood pressure was mildly elevated at $150 / 100 \mathrm{mmHg}$ and normalised without any treatment. The patient complained of acute blurred vision, dizziness, and nausea within the first day of the postpartum period. She did not have any type of headache.

In her neurological examination, eye movements served to maintain all areas; however, she had rotary nystagmus in all directions. Other neurological examinations were normal. She had vertigo but her cerebellar examination was normal.

Diuresis was preserved. The examination of urines by urinary strip was positive $(++++)$. Her haemoglobin value was $10.2 \mathrm{~g} / \mathrm{dL}$ (11.7-15.5 $\mathrm{g} / \mathrm{dL}$ ). On admission, platelet count was 75,000 uL (150-450 uL) and other laboratory results were normal (e.g., aspartate aminotransferase, alanine transaminase, prothrombin time, partial thromboplastin time).

MRI of the brain revealed that there was no diffusion restriction in the diffusion-weighted imaging sequence (Figure $1 \mathrm{~A}$ ), no low signal in the apparent diffusion coefficient map (Figure 1B), T1 weighted (T1W) images showed isointense signals (Figure 1C), and T2W (Figure 1D) and fluid-attenuated inversion recovery (Figure $1 \mathrm{E}$ ) images showed hyperintense signals in the pons during acute period. It presented as vasogenic oedema with no irreversible infarct.

No treatment for PRES lesions was given to the patient, only supportive therapy. One month later a cranial MRI was repeated. MRI results showed that signal abnormalities were no longer present in the pons, in both fluid-attenuated inversion recovery (Figure $2 \mathrm{~A}$ ) and $\mathrm{T} 2$ sequence (Figure 2B). Informed consent was obtained from the patient.

\section{DISCUSSION}

PRES is characterised by headache, confusion, visual disturbances, and seizures. Transient vasogenic oedema occurs predominantly within the posterior circulation regions. ${ }^{2,9}$ PRES with isolated pons involvement has been rarely described..$^{2-7}$ Herein, the authors describe isolated pons involvement in a patient with ITP.

PRES is often related to an acute increase in arterial blood pressure, is clinically indistinguishable from hypertensive encephalopathy, and has been associated with pre-eclampsia, renal failure, infections, immunosuppressive agent use, and hypercalcaemia. ${ }^{2,3}$ In this present case, the authors think that the causes of PRES are mild hypertension and pregnancy. The patient also had mild thrombocytopenia, which may be coincidental or associated with limited disease, but further observations are required to characterise this condition.

Pathophysiology of PRES is not well understood. The current leading theory suggests that hypertension exceeds the limits of autoregulation and causes hyperperfusion, leading to breakdown of the blood-brain barrier and subsequent brain oedema. Because the vertebrobasilar system is sparsely innervated by sympathetic nerves, the posterior brain region is particularly susceptible to breakthrough of autoregulation with elevated blood pressures. Another theory is that an alteration in cerebral vascular tone may lead to vasoconstriction of vessels. Endothelial dysfunction may be important for underlying mechanisms.,10,11 

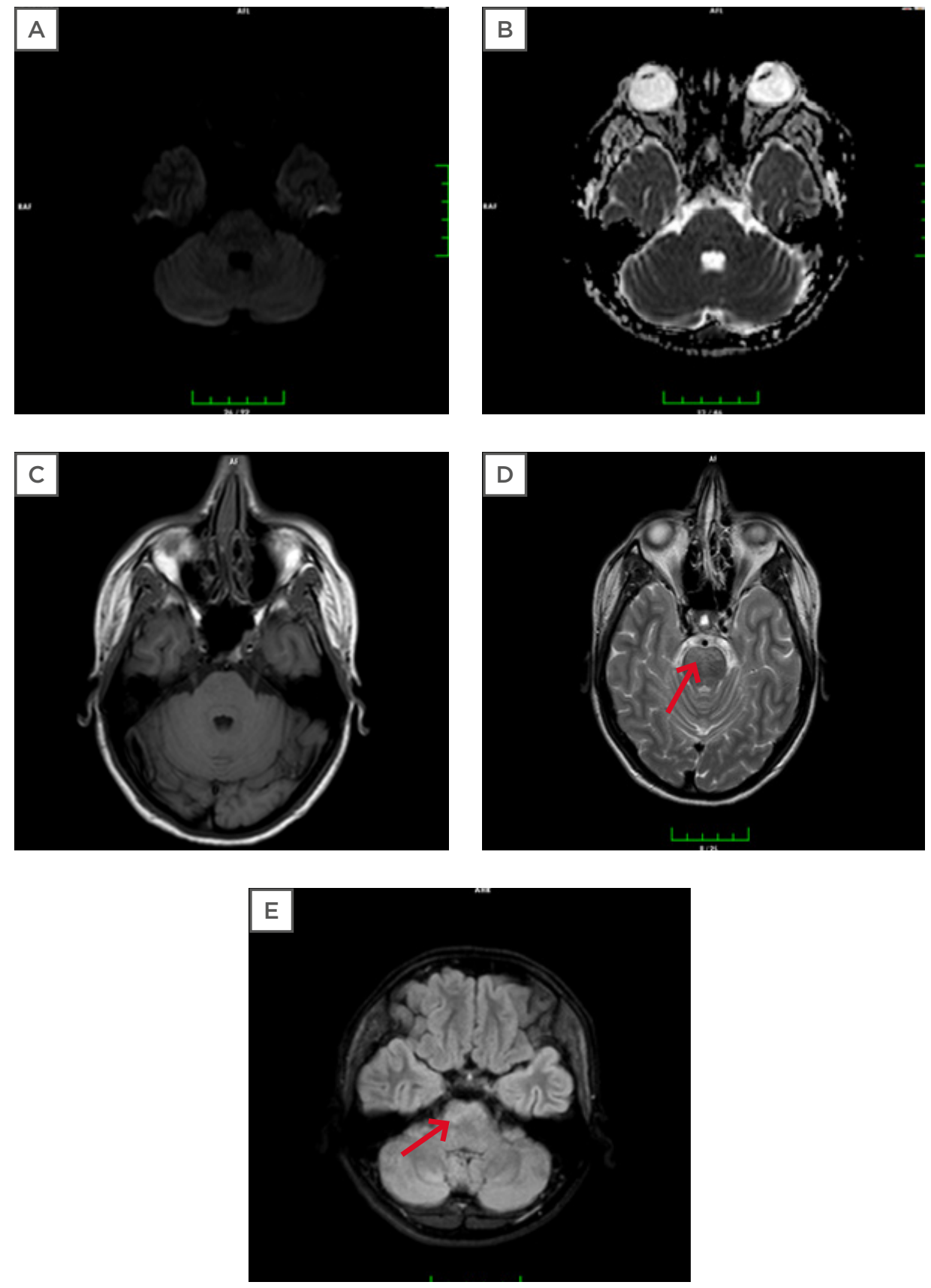

Figure 1: Cranial MRI findings of the patient when they first experienced symptoms.

MRI of the brain revealed that there was (A) no diffusion restriction in the diffusion-weighted imaging sequence; and (B) no low signal in the apparent diffusion coefficient map. (C) T1-weighted images showed isointense signals; (D; arrow) T2 weighted; (E; arrow) fluid-attenuated inversion recovery images showed hyperintense signals in the pons during acute period. It presented as vasogenic oedema with no irreversible infarct.

These theories, however, cannot explain the differences in lesion involvement.

The exact mechanism of PRES is still controversial. Some case reports and reviews of the literature indicate that extraordinarily high pressure is correlated with the development of infratentorial PRES; ${ }^{7,8}$ however, in this case, blood pressure was mildly elevated. The reason of the lesion's limitation may be due to relatively controlled hypertension in that case. However, there are some cases with limited lesions despite uncontrolled hypertension. ${ }^{10}$ 

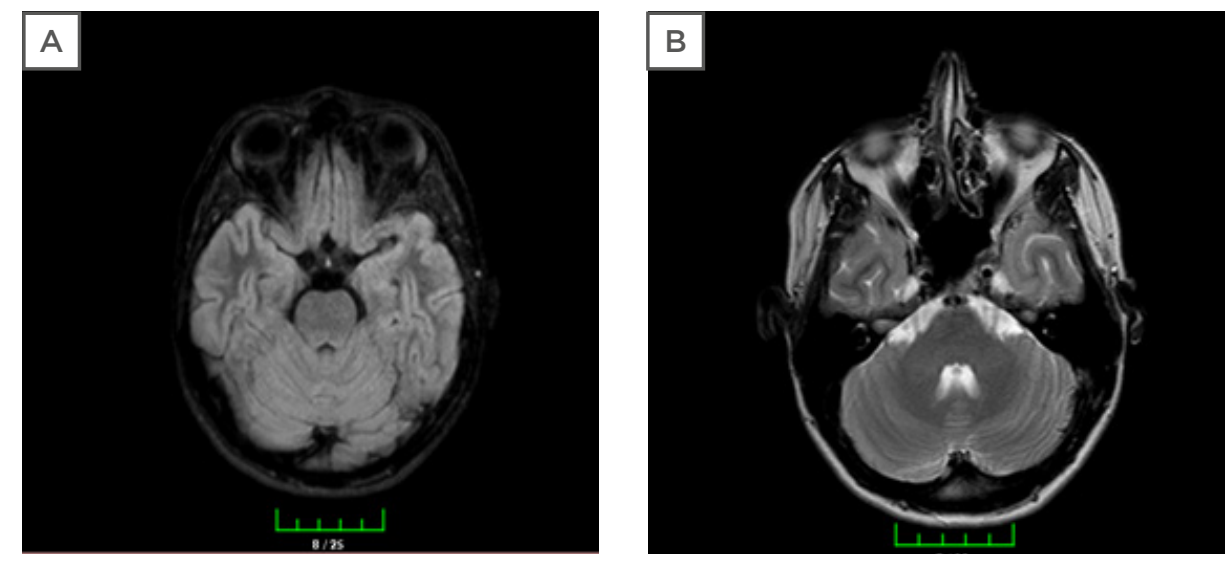

Figure 2: Control cranial MRI results of the patient.

There were no longer signal abnormalities in the pons in (A) fluid-attenuated inversion recovery and (B) T2 sequence.

The authors have hypothesised regarding the pathophysiology of PRES that its mechanism may be associated with a currently unknown type of prolonged spreading depression. Cortical spreading depression is a wave of transient intense neuronal firing leading to a long-lasting depolarising block of neuronal activity.

Cortical spreading depression generated in normoxic tissue does not cause neuronal death, is reversible, and is a proposed pathological mechanism of migraine with aura. ${ }^{12}$ The authors' patient did not have any type of headache before or during PRES symptoms. There are some mechanisms for firing of neurons and reversible depression of posterior areas of the brain which may be related to, amongst others, hypertension, inflammation, and infection. The underlying mechanism for some PRES patients, for example as in this case, who have isolated brainstem involvement without migraine with aura, may be limited cortical depression. There are no studies on this research area. Future studies are required to focus on this issue.

Thrombocytopenia has a number of different aetiologies. ${ }^{13}$ The most common type is pregnancy-associated thrombocytopenia, which accounts for $65-80 \%$ of cases, followed by additional causes such as ITP and hypertensive disorder in pregnancy (pregnancy-induced hypertension $[\mathrm{PIH}])^{14}$ Thrombocytopenia in $\mathrm{PIH}$ is predominantly a result of vascular endothelial ischaemia and hypoxia caused by vascular vasospasm..$^{15}$ However, the authors' patient had ITP before the pregnancy. Blood pressure assessment and regular examination of platelet counts are very important for these patients. Evidence to guide management for pregnant patients with ITP is lacking. ${ }^{13}$

The treatment options for thrombocytopenia in pregnancy are limited. The platelet counts are very important before and after delivery. ${ }^{13}$

There were two different studies that compared the effectiveness of treatment with intravenous Ig compared with corticosteroids treatment; results showed that there were no differences between the two treatment types..$^{13}$ The authors did not administer any kind of treatment to the patient for ITP, and there were no problems related to platelet counts. The differential diagnosis between ITP and thrombocytopenia in PIH is not so easy in some patients. Laboratory evaluations are important before and during pregnancy and during postpartum period. The patient's mild hypertension was treated with nifedipine 60 $\mathrm{mg} /$ day. Because of limited disease, no specific treatment for the disease other than supportive medicines were given to the patient.

The patient in this case had both ITP and PIH, and the authors reported this case for this reason. MRI showed isolated pons involvement. The authors identified only seven cases with isolated pons involvement in PRES. ${ }^{2-7}$ The authors do not know how this disease presentation is possible given that the pathophysiology of this condition is unknown. The authors speculated that the primary causative 
problem was vascular endothelial ischaemia and hypoxia caused by vascular vasospasm and only posterior circulation involvement. Mild hypertension and mild thrombocytopenia may be associated with limited disease involvement. In this case, the authors are unsure of the role of the ITP in generation of PRES; however, they believe that this may be coincidental or associated with limited disease. Further observations are required to characterise the condition.

The differential diagnosis of the brainstem involvement is important because of different treatment modalities. The differential diagnoses include central pontine myelinolysis, brainstem infarction, brainstem glioma, and multiple sclerosis. The main identification of PRES is vasogenic oedema presented in imaging and reversibility of clinic and imaging. ${ }^{7}$ The diagnosis of PRES through MRI relies especially on diffusion-weighted imaging. Isolated pontine involvement in neuroimaging studies are very rare and radiological recovery was observed in all cases. In general, the MRI findings in PRES include T1 signal hypointensity, variable enhancement, and T2 signal hyperintensity, ${ }^{7}$ as seen in the present patient. Whereas the radiological findings of MRI in PRES are resolved within few months, ${ }^{7-8} \mathrm{MRI}$ findings of other diseases such as central pontine myelinolysis and brainstem infarction persist in differential diagnosis. ITP is also a problem for the authors' patient; however, endothelial dysfunction may be important for underlying mechanisms for both clinical conditions.

This case report has some limitations. The authors did not perform a lumbar puncture for cerebrospinal fluid examination for a differential diagnosis. The only hypothesis that the authors have regarding the pathophysiology of the condition does not currently have strong evidence to support it.

\section{KEY TAKE HOME MESSAGES}

PRES may present with brainstem involvement, mild hypertension, and mild thrombocytopenia only in rare cases. This should be evaluated in a differential diagnosis. The authors believe that prolonged spreading depression may have a role in the pathophysiology of PRES. Further studies are needed to support this hypothesis.

\section{References}

1. Ferrara $\mathrm{M}$ et al. Isolated pons involvement in posterior reversible encephalopathy syndrome: case report and review of the literature. NeurologicalSci. 2017;6:51-4.

2. Gao B et al. Isolated pons involvement in posterior reversibile encephalopathy syndrome in patient with chronic renal insufficiency: case report and literature review. Clin Neuroradiol. 2012;22(4):341-4.

3. Cartier $L$ et al. [Pontine reversible leucopathy in an AIDS patient associated with highly active antiretroviral therapy (HAART): report of one case]. Rev Med Chil. 2016 May;144(5):675-9. (In Spanish)

4. Ou S et al. Isolated pons variant of posterior reversible encephalopathy syndrome complicated with ischemic stroke in a young patient. Neurol Sci. 2012;34:1082

5. Tang K. Oxaliplatin-induced posterior reversible encephalopathy syndrome with isoltaed involvement of pons. $J$ Cancer Res Ther. 2015;11(4):1022.

6. Tanioka R. Convalescence of atypical reversible posterior leukoencephalopathy syndrome in human immunodeficiency virus infection. J Med Investig. 2007;54(12):191-4

7. Ou S et al. Posterior reversible encephalopathy syndrome with isolated involving infratentorial structures. Frontier in Neurol. 2018:9:843

8. Samara A et al. Posterior revertsible encephalopathy syndrome with isolated infratentorial involvement: a case report. Radiol Case Rep. 2019;14:576-80.

9. Hinchey $\mathrm{J}$ et al. A reversible posterior leukoencephalopathy syndrome. N Engl J Med. 1996;334:494-500.

10. Chenchen Liu et al. Isolated brainstem involvement in posterior reversible encephalopathy syndrome: a case report and review of the literature. Int J Neurosci. 2019;129(8):808-13.

11. Staykov D, Schwab S. Posterior reversible encephalopathy syndrome. J Intensive Care Med. 2012;27(1):11-24.

12. Desroches $M$ et al. Modeling cortical spreading depression induced by the hyperactivity of interneurons. J Comput Neurosci. 2019;47(2-2):12540.

13. Wang $X$ et al. Thrombocytopenia in pregnancy with different diagnoses differential clinical features treatments and outcomes. Medicine (Baltimore). 2017;96(29):e7561.

14. Gernsheimer T. Thrombocytopenia in pregnancy: is this immune thrombocytopenia or...? Hematology Am Soc Hematol Educ Program. 2012;2012:198-202.

15. Mehta B et al. Hypertension in pregnancy: a community-based study. Indian J Community Med. 2015;40(4):273-8. 